\title{
Streptococcus milleri Group
}

National Cancer Institute

\section{Source}

National Cancer Institute. Streptococcus milleri Group. NCI Thesaurus. Code C86796.

A bacterium that belongs to the Milleri group of the genus Streptococcus. 